EESTI NSV TEADUSTE AKADEEMIA TOIMETISED. XI KOIDE

FOOSIKALIS-MATEMAATILISTE JA TEHNILISTE TEADUSTE SEERIA. 1962, NR. 1

ИЗВЕСТИЯ АКАДЕМИИ НАУК ЭСТОНСКОИ ССР. ТОМ ХІ СЕРИЯ ФИЗИКО-МАТЕМАТИЧЕСКИХ И ТЕХНИЧЕСКИХ НАУК. 1962, $\mathrm{N}_{2} 1$

\title{
О КУСОЧНО-ПОЛИНОМИАЛЬНОЙ АППРОКСИМАЦИИ
}

\author{
И. ПЕТЕРСЕН, \\ кандидат физико-математических наук
}

1. Пусть функция $f(x)$ определена и конечна на отрезке $[a, b]$ и $x_{i}=a+i h$ $\left(i=0,1, \ldots, n, h=\frac{b-a}{n}\right)$ - система равноотстоящих узлов этого отрезка. В статье рассматривается вопрос об интерполировании $f(x)$ функциями, два раза непрерывно дифференцируемыми на $[a, b]$, которые на подинтервалах $x_{i} \leqslant x \leqslant x_{i+1}$ являются алгебраическими полиномами третьей степени. Первое и второе производные этих функций будут в узлах $x_{i}$ выражены через значения функции $f(x)$. Эти выражения можно применять вместо формул численного дифференцирования для случаев, в которых надо пользоваться большим количеством значений функции, причем производных необходимого порядка той же функции не существует. На основании этого спо соба интерполяции построен приближенный метод решения краевых задач для обыкновенных дифференциальных уравнений второго порядка и установлены условия сходимости метода. Раньше такое интерполирование было использовано Д. Голладеем ['] для вывода квадратурной формулы.*

2. Пусть $F[f ; x]$ два раза на $[a, b]$ непрерывно дифференцируемая функция, принимающая в узлах $x_{i}$ значения $y_{i}=f\left(x_{i}\right)$, и такая, что в подинтервале $\left[x_{i}, x_{i+1}\right] \quad(i=0,1, \ldots, n-1) F[f ; x]=\varphi_{i}(x)$ является полиномом третьей степени. Обозначим $\varphi_{i}^{\prime}\left(x_{i}\right)=\varphi_{i}^{\prime}, \varphi_{i}^{\prime \prime}\left(x_{i}\right)=\varphi_{i}^{\prime \prime}, \varphi_{i}^{\prime \prime \prime}\left(x_{i}\right)=\varphi_{i}^{\prime \prime \prime}$.

Тогда в $\left[x_{i}, x_{i+1}\right]$

$$
\begin{aligned}
& \varphi_{i}(x)=y_{i}+\varphi_{i}^{\prime}\left(x-x_{i}\right)+\frac{\varphi_{i}^{\prime \prime}}{2}\left(x-x_{i}\right)^{2}+\frac{\varphi_{i}^{\prime \prime \prime}}{6}\left(x-x_{i}\right)^{3}, \\
& \varphi_{i}^{\prime}(x)=\varphi_{i}^{\prime}+\varphi_{i}^{\prime \prime}\left(x-x_{i}\right)+\frac{\varphi_{i}^{\prime \prime \prime}}{2}\left(x-x_{i}\right)^{2}, \\
& \varphi_{i}^{\prime \prime}(x)=\varphi_{i}^{\prime \prime}+\varphi_{i}^{\prime \prime \prime}\left(x-x_{i}\right) .
\end{aligned}
$$

Вследствие непрерывности и непрерывной дифференцируемости функцни $F[f ; x]$ до второго порядка, из (2.1) получим

* Уже после сдачи настоящей статьн в печать автору стали известны еще три статьи, посвященные этой проблеме: I. J. S c ho en be r g, Quart. Appl. Math., 4, 1946, 45-99 u 112-141; G. Birk of f, H. I. Ga rabed i a n, J. Math. Physics, 39, 1960, 258-268. 


$$
\begin{aligned}
& y_{i+1}=y_{i}+\varphi_{i}^{\prime} h+\frac{\varphi_{i}^{\prime \prime}}{2} h^{2}+\frac{\varphi_{i}^{\prime \prime}}{6} h^{3}, \\
& \varphi_{i+1}^{\prime}=\varphi_{i}^{\prime}+\varphi_{i}^{\prime \prime} h+\frac{\varphi_{i}^{\prime \prime}}{2} h^{2}, \\
& \varphi_{i+1}^{\prime \prime}=\varphi_{i}^{\prime \prime}+\varphi_{i}^{\prime \prime \prime} h
\end{aligned}
$$

и после исключения $\varphi_{i}^{\prime \prime}$

$$
\begin{aligned}
& \varphi_{i+1}^{\prime}=-2 \varphi_{i}^{\prime}-\frac{h}{2} \varphi_{i}^{\prime \prime}+\frac{3}{h}\left(y_{i+1}-y_{i}\right), \\
& \varphi_{i+1}^{\prime \prime}=-\frac{6}{h} \varphi_{i}^{\prime}-2 \varphi_{i}^{\prime \prime}+\frac{6}{h^{2}}\left(y_{i+1}-y_{i}\right) .
\end{aligned}
$$

Обозначив

$$
\overrightarrow{u_{i}}=\left\|\begin{array}{c}
\varphi_{i}^{\prime} \\
\varphi_{i}^{\prime \prime}
\end{array}\right\|, \quad D=\left\|\begin{array}{lr}
-2 & -\frac{h}{2} \\
-\frac{6}{h} & -2
\end{array}\right\|, \quad \vec{v}=\left\|\begin{array}{c}
\frac{3}{h} \\
\frac{6}{h^{2}}
\end{array}\right\|,
$$

(2.3) принимает вид

$$
\vec{u}_{i+1}=D \overrightarrow{u_{i}}+\left(y_{i+1}-y_{i}\right) \vec{v}
$$

так что

$$
\overrightarrow{u_{i}}=D \overrightarrow{i u_{0}}+\left[\left(y_{1}-y_{0}\right) D^{i-1}+\ldots+\left(y_{i-1}-y_{i-2}\right) D+\left(y_{i}-y_{i-1}\right) E\right] \vec{v}
$$

или

$$
\begin{aligned}
& \overrightarrow{u_{i}}=D^{i} u_{0}-y_{0} D^{i-1} \vec{v}+y_{1}(D-E) D^{i-2} \vec{v}+\ldots+y_{i-2}(D-E) D \vec{v}+ \\
& +y_{i-1}(D-E) \vec{v}+y_{i} \vec{v}
\end{aligned}
$$

Если $-\lambda_{1}$ и $-\lambda_{2}$ собственные значения матрицы $D$, то

$$
\lambda_{1}=2+\sqrt{3}, \quad \lambda_{2}=2-\sqrt{3}
$$

ห

$$
\begin{aligned}
& D^{i}=(-1)^{i} \mid \begin{array}{lc}
\frac{\lambda_{1}^{i}+\lambda_{2}^{i}}{2} & \frac{\lambda_{1}^{i}-\lambda_{2}^{i}}{4 \sqrt{3}} h \\
\frac{\lambda_{1}^{i}-\lambda_{2}^{i}}{h} \sqrt{3} & \frac{\lambda_{1}^{i}+\lambda_{2}}{2}
\end{array} \|, \\
& D^{i-1} \vec{v}=(-1)^{i-1}(3-\sqrt{3})\left\|\begin{array}{l}
\frac{\lambda_{1}^{i}+\lambda_{2}^{i-1}}{2 h} \\
\frac{\lambda_{1}^{i}-\lambda_{2}^{i-1}}{h^{2}} \sqrt{3}
\end{array}\right\| \\
& (D-E) \overrightarrow{D^{k v}}=(-1)^{k+1}\left|\begin{array}{l}
\frac{\lambda_{1}^{k+1}+\lambda_{2}^{k+1}}{h} 3 \\
\frac{\lambda_{1}^{k+1}-\lambda_{2}^{k+1}}{h^{2}} 6 \sqrt{3}
\end{array}\right|
\end{aligned}
$$


Из (2.4) получается при помощи (2.6)

$$
\begin{aligned}
& \varphi_{i}^{\prime}=(-1)^{i}\left[\frac{1}{2}\left(\lambda_{1}^{i}+\lambda_{2}^{i}\right) \varphi_{0}^{\prime}+\frac{h}{4 \sqrt{3}}\left(\lambda_{1}^{i}-\lambda_{2}^{i}\right) \varphi_{0}^{\prime \prime}+\frac{3-\sqrt{3}}{2 h}\left(\lambda_{1}^{i}+\lambda_{2}^{i-1}\right) y_{0}+\right. \\
&+\left.\frac{3}{h} \sum_{k=1}^{i-1}(-1)^{k}\left(\lambda_{1}^{i}-k+\lambda_{2}^{i-k}\right) y_{k}+\frac{3(-1)^{i}}{h} y^{i}\right], \\
& \varphi_{i}^{\prime \prime}=(-1)^{i}\left[\frac{\sqrt{3}}{h}\left(\lambda_{1}^{i}-\lambda_{2}^{i}\right) \varphi_{0}^{\prime}+\frac{1}{2}\left(\lambda_{1}^{i}+\lambda_{2}^{i}\right) \varphi_{0}^{\prime \prime}+\frac{3 \sqrt{3}-3}{h^{2}}\left(\lambda_{1}^{i}-\lambda_{2}^{i-1}\right) y_{0}+\right. \\
&\left.+\frac{6 \sqrt{3}}{h^{2}} \sum_{k=1}^{i-1}(-1)^{k}\left(\lambda_{1}^{i-k}-\lambda_{2}^{i-k}\right) y_{k}+\frac{6(-1)^{i}}{h^{2}} y_{i}\right] .
\end{aligned}
$$

Для однозначного определения $\varphi_{i}^{\prime}, \varphi_{i}^{\prime \prime}$ и тем самым $F[f ; x]$ придется, кроме значений функции $f(x)$ в узлах, задавать еще и некоторые «краевые» или «начальные» условия, позволяющие в (2.7) определить $\varphi_{0}^{\prime}$ и $\varphi_{0}^{\prime \prime}$. Рассмотрим три таких условия «краевого» типа.

При $F[f ; x]=F_{1}[; ; x]$ потребуем, чтобы

$$
F_{\mathrm{I}}^{\prime}\left[f ; x_{0}\right]=y_{0}^{\prime}, \quad F_{\mathrm{I}}^{\prime}\left[f ; x_{n}\right]=y_{n}^{\prime},
$$

при $F_{\text {II }}[f ; x]$, чтобы*

$$
F_{\text {II }}^{\prime \prime}\left[f ; x_{0}\right]=F_{11}^{\prime \prime}\left[f ; x_{n}\right]=0 \text {, }
$$

и $F_{\mathrm{III}}[f ; x]$ определяем при помощи условий

$$
F_{1 I I}\left[f ; x_{1 / 2}\right]=y_{1 / 2}, F_{1 I I}\left[f ; x_{n-1 / 2}\right]=y_{n-1 / 2} .
$$

Из (2.8) и первого из равенств (2.7) при $i=n$ получаем выражение для $\varphi_{0}^{\prime \prime}$ и устанавливаем, что $\varphi_{0}^{\prime}=y_{0}^{\prime}$. После подстановки их в $(2.7)$ имеем для случая $F_{\mathrm{I}}[f ; x]$

$$
\begin{aligned}
& \varphi_{1, i}^{\prime}= \frac{3(-1)^{i}}{h\left(\lambda_{1}^{n}-\lambda_{2}^{n}\right)}\left[\left(\lambda_{1}^{n-i}-\lambda_{2}^{n-i}\right)\left(\frac{h}{3} y_{0}^{\prime}+y_{0}+\sum_{k=1}^{i-1}(-1)^{k}\left(\lambda_{1}^{k}+\lambda_{2}^{k}\right) y_{k}\right)-\right. \\
&-(-1)^{i}\left(\lambda_{1}^{i} \lambda_{2}^{n-i}-\lambda_{1}^{n-i} \lambda_{-2}^{i}\right) y_{i}-\left(\lambda_{1}^{i}-\lambda_{2}^{i}\right)\left(\sum_{k=i+1}^{n-1}(-1)^{k}\left(\lambda_{1}^{n-k}+\lambda_{2}^{n-k}\right) y_{k}+\right. \\
&\left.\left.+(-1)^{n} y_{n}-\frac{h}{3}(-1)^{n} y_{n}^{\prime}\right)\right], \\
& \varphi_{1, i}^{\prime \prime}=\frac{6 \sqrt{3}(-1)^{i+1}}{h^{2}\left(\lambda_{1}^{n}-\lambda_{2}^{n}\right)}\left[\left(\lambda_{1}^{n-i}+\lambda_{2}^{n-i}\right)\left(\frac{h}{3} y_{0}^{\prime}+y_{0}+\sum_{k=1}^{i-1}(-1)^{k}\left(\lambda_{1}^{k}+\lambda_{2}^{k}\right) y_{k}\right)+\right. \\
&+(-1)^{i}\left(\frac{3+\sqrt{3}}{3}\left(\lambda_{1}^{n-1}+\lambda_{2}^{n}\right)+\lambda_{1}^{i} \lambda_{2}^{n-i}+\lambda_{1}^{n-i} \lambda_{2}^{i}\right) y_{i}+
\end{aligned}
$$




$$
\left.+\left(\lambda_{1}^{i}+\lambda_{2}^{i}\right)\left(\sum_{k=i+1}^{n-1}(-1)^{k}\left(\lambda_{1}^{n-k}+\lambda_{2}^{n-k}\right) y_{k}+(-1)^{n} y_{n}-\frac{h}{3}(-1)^{n} y_{n}^{\prime}\right)\right]
$$

при $i=1,2, \ldots, n-1$ и

$$
\begin{aligned}
& \varphi_{1,0}^{\prime}=y_{0}^{\prime}, \quad \varphi_{1, n}^{\prime}=y_{n}^{\prime}, \\
& \varphi_{1,0}^{\prime \prime}=\frac{-6 \sqrt{3}}{h^{2}\left(\lambda_{1}^{n}-\lambda_{2}^{n}\right)}\left[\frac{h}{3}\left(\lambda_{1}^{n}+\lambda_{2}^{n}\right) y_{0}^{\prime}+\frac{3+\sqrt{3}}{3}\left(\lambda_{1}^{n-1}+\lambda_{2}^{n}\right) y_{0}+\right. \\
& \left.+2\left(\sum_{k=1}^{n-1}(-1)^{k}\left(\lambda_{1}^{n-k}+\lambda_{2}^{n-k}\right) y_{k}+(-1)^{n} y_{n}-\frac{h}{3}(-1)^{n} y_{n}^{\prime}\right)\right], \\
& \varphi_{1, n}^{\prime \prime}=\frac{6 \sqrt{3}(-1)^{n+1}}{h^{2}\left(\lambda_{1}^{n}-\lambda_{2}^{n}\right)}\left[2\left(\frac{h}{3} y_{0}^{\prime}+y_{0}+\sum_{k=1}^{n-1}(-1)^{k}\left(\lambda_{1}^{k}+\lambda_{2}^{k}\right) y_{k}\right)+\right. \\
& \left.+\frac{3+\sqrt{3}}{3}(-1)^{n}\left(\lambda_{1}^{n-1}+\lambda_{2}^{n}\right) y_{n}-\frac{h}{3}(-1)^{n}\left(\lambda_{1}^{n}+\lambda_{2}^{n}\right) y_{n}^{\prime}\right] .
\end{aligned}
$$

Соответствующие формулы для $F_{\mathrm{II}}[f ; x]$ принимают вид $(i=0,1, \ldots, n)$ :

$$
\varphi_{\mathrm{II}, i}^{\prime}=\frac{3(-1)^{i}}{h\left(\lambda_{1}^{n}-\lambda_{2}^{n}\right)}\left[\left(\lambda_{1}^{n-i}+\lambda_{2}^{n-i}\right)\left(\frac{1}{\sqrt{3}} y_{0}+\sum_{k=1}^{i-1}(-1)^{k}\left(\lambda_{1}^{k}-\lambda_{2}^{k}\right) y_{k}\right)+\right.
$$

$\left.+(-1)^{i}\left(\lambda_{1}^{i} \lambda_{2}^{n-i}-\lambda_{1}^{n-i} \lambda_{2}^{i}\right) y_{i^{--}}\left(\lambda_{1}^{i}+\lambda_{2}^{i}\right)\left(\sum_{k=i+1}^{n-1}(-1)^{k}\left(\lambda_{1}^{n-k}-\lambda_{2}^{n-k}\right) y_{k}+\frac{1}{\sqrt{3}}(-1)^{n} y_{n}\right)\right]$,

$$
\begin{aligned}
\varphi_{\mathrm{II}, i}^{\prime \prime}= & \frac{6 \sqrt{3}(-1)^{i+1}}{h^{2}\left(\lambda_{1}^{n}-\lambda_{2}^{n}\right)}\left[\left(\lambda_{1}^{n-i}-\lambda_{2}^{n-i}\right)\left(\frac{1}{\sqrt{3}} y_{0}+\sum_{k=1}^{i-1}(-1)^{k}\left(\lambda_{1}^{k}-\lambda_{3}^{k}\right) y_{k}\right)+\right. \\
& +(-1)^{i}\left(\frac{3+\sqrt{3}}{3}\left(\lambda_{1}^{n-i}+\lambda_{2}^{n}\right)-\lambda_{1}^{i} \lambda_{2}^{n-i}-\lambda_{1}^{n-i} \lambda_{2}^{i}\right) y_{i}+ \\
& \left.+\left(\lambda_{1}^{i}-\lambda_{2}^{i}\right)\left(\sum_{k=i+1}^{n-1}(-1)^{k}\left(\lambda_{1}^{n-k}-\lambda_{2}^{n-k}\right) y_{k}+\frac{1}{\sqrt{3}}(-1)^{n} y_{n}\right)\right]
\end{aligned}
$$

Наконец, получаем формулы для $\varphi_{\text {III, } i}$ и $\varphi_{\text {III, } i}$, нспользуя формулы, аналогичные (2.7), и соотношения

$$
\begin{aligned}
6 h \varphi_{\mathrm{III}, 1}^{\prime}-h^{2} \varphi_{\mathrm{III}, 1}^{\prime \prime} & =2 y_{0}-16 y_{1 / 2}+14 y_{1}, \\
6 h \varphi_{\mathrm{III}, n-1}+h^{2} \varphi_{\mathrm{III}, n-1}^{\prime \prime} & =-14 y_{n-1}+16 y_{n-1 / 2}-2 y_{n},
\end{aligned}
$$

которые получаются из условий, что $y=\varphi_{\mathrm{II}, 0}(x)$ проходит через точки $\left(x_{0} ; y_{0}\right)$, $\left(x_{1 / 2} ; y_{1 / 2}\right),\left(x_{1} ; y_{1}\right)$ и $y=\varphi_{111, n}(x)$ - через точки $\left(x_{n-1} ; y_{n-1}\right),\left(x_{n-1 / 2} ; y_{n-1 / 2}\right)$, $\left(x_{n} ; y_{n}\right)$. 
Имеем для $i=2,3, \ldots, n-2$ :

$$
\begin{gathered}
\varphi_{\mathrm{III}, i}^{\prime}=\frac{3(-1)^{i}}{h\left(\lambda_{1}^{n-1}-\lambda_{2}^{n-1}\right)}\left[-\frac{3+\sqrt{3}}{18}\left(\lambda_{1}^{n-i-1}-\lambda_{2}^{n-i}\right)\left(y_{0}-8 y_{1 / 2}+19 y_{1}\right)+\right. \\
+\left(\lambda_{1}^{n-i-1}-\lambda_{2}^{n-i}\right) \sum_{k=2}^{i-1}(-1)^{k}\left(\lambda_{1}^{k}+\lambda_{2}^{k-1}\right) y_{k}-(-1)^{i}\left(\lambda_{1}^{i} \lambda_{2}^{n-i}-\lambda_{1}^{n-i} \lambda_{2}^{i}\right) y_{i}-\quad(2.19) \\
-\left(\lambda_{1}^{i-1}-\lambda_{2}^{i}\right) \sum_{k=i+1}^{n-2}(-1)^{k}\left(\lambda_{1}^{n-k}+\lambda_{2}^{n-k-1}\right) y_{k}+(-1)^{n} \frac{3+\sqrt{3}}{18}\left(\lambda_{1}^{i-1}-\lambda_{2}^{i}\right)\left(19 y_{n-1}-\right. \\
\varphi_{\mathrm{III}, i}^{n}=\frac{6 \sqrt{3}(-1)^{i}}{h^{2}\left(\lambda_{1}^{n-1}-\lambda_{2}^{n-1}\right)}\left[\frac{3+\sqrt{3}}{18}\left(\lambda_{1}^{n-i-1}+\lambda_{2}^{n-i}\right)\left(y_{0}-8 y_{1 / 2}+19 y_{1}\right)+\right. \\
+\left(\lambda_{1}^{n-i-1}+\lambda_{2}^{n-i}\right) \sum_{k=2}^{i-1}(-1)^{k}\left(\lambda_{1}^{k}+\lambda_{2}^{k-1}\right) y_{k}-(-1)^{i}\left(\frac{3+\sqrt{3}}{3}\left(\lambda_{1}^{n-2}+\lambda_{2}^{n-1}\right)+\right. \\
\left.+\lambda_{1}^{i} \lambda_{2}^{n-i}+\lambda_{1}^{n-i} \lambda_{2}^{i}\right) y_{i}-\left(\lambda_{1}^{i-1}+\lambda_{2}^{i}\right) \sum_{k=i+1}^{n-2}(-1)^{k}\left(\lambda_{1}^{n-k}+\lambda_{2}^{n-k-1}\right) y_{k}+ \\
\left.+(-1)^{n} \frac{3+\sqrt{3}}{18}\left(\lambda_{1}^{i-1}+\lambda_{2}^{i}\right)\left(19 y_{n}-8 y_{n-1 / 2}+y_{n}\right)\right]
\end{gathered}
$$

н

$$
\varphi_{\mathrm{III}, 0}^{\prime}=\frac{3}{h\left(\lambda_{1}^{n-1}-\lambda_{2}^{n-1}\right)}\left\{-\frac{1}{18}\left[(21+\sqrt{3}) \lambda_{1}^{n-1}-(21-\sqrt{3}) \lambda_{2}^{n-1}\right] y_{0}+\right.
$$

$+\frac{4}{9}(3+\sqrt{3})\left(\lambda_{1}^{n-1}-\lambda_{2}^{n}\right) y_{1 / 2}+\frac{1}{18}\left[(15-19 \sqrt{3}) \lambda_{1}^{n-1}-(15+19 \sqrt{3}) \lambda_{2}^{n-1}\right] y_{1}-$

$\left.-(1-\overline{\sqrt{3}}) \sum_{k=2}^{n-2}(-1)^{k}\left(\lambda_{1}^{n-k}+\lambda_{2}^{n-k-1}\right) y_{k}-(-1)^{n} \frac{\sqrt{3}}{9}\left(19 y_{n-1}-8 y_{n-1 / 2}+y_{n}\right)\right\}$

$\varphi_{\mathrm{III}, 1}^{\prime}=\frac{-3}{h\left(\lambda_{1}^{n-1}-\lambda_{2}^{n-1}\right)}\left\{-\frac{3+\sqrt{3}}{18}\left(\lambda_{1}^{n-2}-\lambda_{2}^{n-1}\right)\left(y_{0}-8 y_{1 / 2}\right)+\frac{\sqrt{3}}{18}\left[(1+7 \sqrt{3}) \lambda_{1}^{n-2}+\right.\right.$

$$
\left.+(1-7 \sqrt{3}) \lambda_{2}^{n-2}\right] y_{1}+(1-\sqrt{3}) \sum_{k=2}^{n-2}(-1)^{k}\left(\lambda_{1}^{n-k}+\lambda_{2}^{n-k-1}\right) y_{k}+
$$

$$
\left.+(-1)^{n} \frac{\sqrt{3}}{9}\left(19 y_{n-1}-8 y_{n-1 / 2}+y_{n}\right)\right\}
$$

$$
\varphi_{\mathrm{III}, 0}^{\prime \prime}=\frac{6 \sqrt{3}}{h^{2}\left(\lambda_{1}^{n-1}-\lambda_{2}^{n-1}\right)}\left\{\frac{1}{18}\left[(3+7 \sqrt{3}) \lambda_{1}^{n-1}+(3-7 \sqrt{3}) \lambda_{2}^{n-1}\right] y_{0}-\right.
$$

$-\frac{4}{3}(3+\sqrt{3})\left(\lambda_{1}^{n-1}+\lambda_{2}^{n}\right) y_{1 / 2}+\frac{1}{18}\left[(57-17 \sqrt{3}) \lambda_{1}^{n-1}+(57+17 \sqrt{3}) \lambda_{2}^{n-1}\left[y_{1}-(2.23)\right.\right.$

$$
\left.-(3-\sqrt{3}) \sum_{k=2}^{n-2}(-1)^{k}\left(\lambda_{1}^{n-k}+\lambda_{-2}^{n-k-1}\right) y_{k}+(-1)^{n} \frac{1}{3}\left(19 y_{n-1}-8 y_{n-1 / 2}+y_{n}\right)\right\}
$$




$$
\begin{aligned}
& \varphi_{\mathrm{III}, 1}^{\prime \prime}= \frac{-6 \sqrt{3}}{h^{2}\left(\lambda_{1}^{n-1}-\lambda_{2}^{n-1}\right)}\left\{\frac{3+\sqrt{3}}{18}\left(\lambda_{1}^{n-2}+\lambda_{2}^{n-1}\right)\left(y_{0}-8 y_{1 / 2}\right)+\right. \\
&+\frac{1}{18}\left[(39+7 \sqrt{3}) \lambda_{1}^{n-2}+(39-7 \sqrt{3}) \lambda_{2}^{n-2}\right] y_{1}- \\
&\left.-(3-\sqrt{3}) \sum_{k=2}^{n-2}(-1)^{k}\left(\lambda_{1}^{n-k}+\lambda_{2}^{n-k-1}\right) y_{k}+(-1)^{n} \frac{1}{3}\left(19 y_{n-1}-8 y_{n-1 / 3}+y_{n}\right)\right\} .
\end{aligned}
$$

Перенумерацией $y$-ов и изменением знака у $h$ из последних четырех формул получаются соответствующие выражения и для $\varphi_{\mathrm{III}, n-1}^{\prime}, \varphi_{\mathrm{III}, n}^{\prime}, \varphi_{\mathrm{III}, n-1}^{\prime \prime}$ и $\varphi_{\mathrm{III}, n}^{\prime \prime}$.

Отметим, что все приведенные формулы линейны и имеют рациональные коэффициенты.

Выпишем в качестве примера эти формулы для $n=5$ :

$\varphi_{\mathrm{I}, 0}^{\prime}=y_{0}^{\prime}$

$\varphi_{\mathrm{I}, 1}^{\prime}=\frac{1}{209 h}\left(-56 h y_{0}^{\prime}-168 y_{0}+45 y_{1}+156 y_{2}-42 y_{3}+12 y_{4}-3 y_{5}+h y_{5}^{\prime}\right)$

$\varphi_{\mathrm{I}, 2}^{\prime}=\frac{1}{209 h}\left(15 h y_{0}^{\prime}+45 y_{0}-180 y_{1}+3 y_{2}+168 y_{3}-48 y_{4}+12 y_{5}-4 h y_{5}^{\prime}\right)$

$\varphi_{\mathrm{I}, 3}^{\prime}=\frac{1}{209 h}\left(-4 h y_{0}^{\prime}-12 y_{0}+48 y_{1}-168 y_{2}-3 y_{3}+180 y_{4}-45 y_{5}+15 h y_{5}^{\prime}\right)$

$\dot{\varphi_{1,4}^{\prime}}=\frac{1}{209 h}\left(h y_{0}^{\prime}+3 y_{0}-12 y_{1}+42 y_{2}-156 y_{3}-45 y_{4}+168 y_{5}-56 h y_{5}^{\prime}\right)$

$\varphi_{\mathrm{I}, 5}^{\prime}=y_{5}$

$\varphi_{I, 0}^{\prime \prime}=\frac{1}{209 h^{2}}\left(-724 h y_{0}^{\prime}-918 y_{0}+1164 y_{1}-312 y_{2}^{\prime}+84 y_{3}-24 y_{4}+6 y_{5}-2 h y_{5}^{\prime}\right)$

$\varphi_{\mathrm{I}, 1}^{\prime \prime}=\frac{1}{209 h^{2}}\left(194 h y_{0}^{\prime}+582 y_{0}-1074 y_{1}+624 y_{2}-168 y_{3}+48 y_{4}-12 y_{5}+4 h y_{5}^{\prime}\right)$

$\varphi_{I, 2}^{\prime \prime}=\frac{1}{209 h^{2}}\left(-52 h y_{0}^{\prime}-156 y_{0}+624 y_{1}-930 y_{2}+588 y_{3}-168 y_{4}+42 y_{5}-14 h y_{5}^{\prime}\right)$

$\varphi_{I, 3}^{\prime \prime}=\frac{1}{209 h^{2}}\left(14 h y_{0}^{\prime}+42 y_{0}-168 y_{1}+588 y_{2}-930 y_{3}+624 y_{4}-156 y_{5}+52 h y_{5}^{\prime}\right)$

$\varphi_{1,4}^{\prime \prime}=\frac{1}{209 h^{2}}\left(-4 h y_{0}^{\prime}-12 y_{0}+48 y_{1}-168 y_{2}+624 y_{3}-1074 y_{4}+582 y_{5}-194 h y_{5}^{\prime}\right)$

$\varphi_{\mathrm{I}, 5}^{\prime \prime}=\frac{1}{209 h^{2}}\left(2 h y_{0}^{\prime}+6 y_{0}-24 y_{1}+84 y_{2}-312 y_{3}+1164 y_{4}-918 y_{5}+724 h y_{5}^{\prime}\right)$

$\varphi_{\mathrm{II}, 0}^{\prime}=\frac{1}{209 h}\left(-265 y_{0}+336 y_{1}-90 y_{2}+24 y_{3}-6 y_{4}+y_{5}\right)$

$\dot{\varphi}_{\mathrm{II}, 1}^{\prime}=\frac{1}{209 h}\left(-97 y_{0}-45 y_{1}+180 y_{2}-48 y_{3}+12 y_{4}-2 y_{5}\right)$

$\dot{\varphi_{\mathrm{II}, 2}}=\frac{1}{209 h}\left(26 y_{0}-156 y_{1}-3 y_{2}+168 y_{3}-42 y_{4}+7 y_{\mathrm{s}}\right)$

$\varphi_{\mathrm{II}, 3}^{\prime}=\frac{1}{209 h}\left(-7 y_{0}+42 y_{1}-168 y_{2}+3 y_{3}+156 y_{4}-26 y_{5}\right)$

$\varphi_{\mathrm{II}, 4}^{\prime}=\frac{1}{209 h}\left(2 y_{0}-12 y_{1}+48 y_{2}-180 y_{3}+45 y_{4}+97 y_{5}\right)$

$\dot{\varphi_{\mathrm{II}, 5}}=\frac{1}{209 \vec{h}}\left(-y_{0}+6 y_{1}-24 y_{2}+90 y_{3}-336 y_{4}+265 y_{5}\right)$

$\varphi_{\mathrm{II}, 0}^{\prime \prime}=0$

$\varphi_{\mathrm{II}, 1}^{\prime \prime}=\frac{1}{209 h^{2}}\left(336 y_{0}-762 y_{1}+540 y_{2}-144 y_{3}+36 y_{4}-6 y_{5}\right)$

$\varphi_{\mathrm{II}, 2}^{\prime \prime}=\frac{1}{209 h^{2}}\left(-90 y_{0}+540 y_{1}-906 y_{2}+576 y_{3}-144 y_{4}+24 y_{5}\right)$

$\varphi_{\mathrm{II}, 3}^{\prime \prime}=\frac{1}{209 h^{2}}\left(24 y_{0}-144 y_{1}+576 y_{2}-906 y_{3}+540 y_{4}-90 y_{5}\right)$

$\varphi_{\mathrm{II}, 4}^{\prime \prime}=\frac{1}{209 h^{2}}\left(-6 y_{0}+36 y_{1}-144 y_{2}+540 y_{3}-762 y_{4}+336 y_{5}\right)$

$\varphi_{\mathrm{II}, 5}^{\prime \prime}=0$. 


$$
\begin{aligned}
& \varphi_{\mathrm{III}, 0}^{\prime}=\frac{1}{336 h}\left(-1273 y_{0}+2120 y_{1 / 2}-1003 y_{1}+198 y_{2}-54 y_{3}+19 y_{4}-8 y_{41 / 2}+y_{5}\right) \\
& \varphi_{\mathrm{III}, 1}^{\prime}=\frac{1}{336 h}\left(71 y_{0}-568 y_{1 / 2}+341 y_{1}+198 y_{2}-54 y_{3}+19 y_{4}-8 y_{41 / 2}+y_{5}\right) \\
& \varphi_{\mathrm{III}, 2}=\frac{1}{336 h}\left(-19 y_{0}+152 y_{1 / 2}-361 y_{1}+18 y_{2}+270 y_{3}-95 y_{4}+40 y_{41 / 2}-5 y_{5}\right) \\
& \dot{\varphi}_{\mathrm{III}, 3}^{\prime}=\frac{1}{336 h}\left(5 y_{0}-40 y_{1 / 2}+95 y_{1}-270 y_{2}-18 y_{3}+361 y_{4}-152 y_{41 / 2}+19 y_{5}\right) \\
& \varphi_{\mathrm{III}, 4}^{\prime}=\frac{1}{336 h}\left(-y_{0}+8 y_{1 / 2}-19 y_{1}+54 y_{2}-198 y_{3}-341 y_{4}+568 y_{41 / 2}-71 y_{5}\right) \\
& \varphi_{\mathrm{III}, 5}^{\prime}=\frac{1}{336 h}\left(-y_{0}+8 y_{1 / 2}-19 y_{1}+54 y_{2}-198 y_{3}+1003 y_{4}-2120 y_{41 / 2}+1273 y_{5}\right) \\
& \varphi_{\mathrm{III}, \theta}^{\prime \prime}=\frac{1}{56 h^{2}}\left(489 y_{0}-1224 y_{1 / 2}+891 y_{1}-198 y_{2}+54 y_{3}-19 y_{4}+8 y_{41 / 2}-y_{5}\right) \\
& \varphi_{\mathrm{III}, 1}^{\prime \prime}=\frac{1}{56 h^{2}}\left(-41 y_{0}+328 y_{1 / 2}-443 y_{1}+198 y_{2}-54 y_{3}+19 y_{4}-8 y_{41 / 2}+y_{5}\right) \\
& \varphi_{\mathrm{III}, 2}^{\prime \prime}=\frac{1}{112 h^{2}}\left(11 y_{0}-88 y_{1 / 2}+209 y_{1}-258 y_{2}+162 y_{3}-57 y_{4}+24 y_{41 / 2}-3 y_{5}\right) \\
& \varphi_{\mathrm{III}, 3}^{\prime \prime}=\frac{1}{112 h^{2}}\left(-3 y_{0}+24 y_{1 / 2}-57 y_{1}+162 y_{2}-258 y_{3}+209 y_{4}-88 y_{41 / 2}+11 y_{5}\right) \\
& \varphi_{\mathrm{III}, 4}^{\prime \prime}=\frac{1}{56 h^{2}}\left(y_{0}-8 y_{1 / 2}+19 y_{1}-54 y_{2}+198 y_{3}-443 y_{4}+328 y_{41 / 2}-41 y_{5}\right) \\
& \varphi_{\mathrm{III}, 5}^{\prime \prime}=\frac{1}{56 h^{2}}\left(-y_{0}+8 y_{1 / 2}-19 y_{1}+54 y_{2}-198 y_{3}-891 y_{4}+1224 y_{41 / 2}-489 y_{5}\right)
\end{aligned}
$$

Исключая из $(2.1)$ и (2.2) $\varphi_{i}^{\prime \prime}$, получим при помощи формул $(2.11)-(2.24)$ явные выражения для $F_{\mathrm{I}}[f ; x], F_{\mathrm{II}}[f ; x]$ и $F_{\mathrm{III}}[f ; x]$ и для их пронзводных. Формулы (2.11)-(2.24) можно рассматривать и самостоятельно как формулы приближенного дифференцирования, принимая $f^{\prime}\left(x_{i}\right) \approx \varphi_{i}^{\prime}, f^{\prime \prime}\left(x_{i}\right) \approx \varphi_{i}^{\prime \prime}$. В ['] показано, что рассмотрецный способ аппроксимации можно в первом приближении механически интерпретировать как замену кривой $y=f(x)$ упругим стержнем, укрепленным в точках $\left(x_{i} ; y_{i}\right)$.

3. Рассмотрим применение полученных формул для приближенного решения дифференцнального уравнения

$$
y^{\prime \prime}+p(x) y^{\prime}+q(x) y=r(x)
$$

гри граничных условиях

$$
y(a)=y(b)=0
$$

Для конкретности ограничимся вариантом III из 2.

Заменяем в (3.1) $y$ и ее пронзводные соответственно через $F_{\text {III }}[f ; x]$ и ее производными н потребуем, чтобы полученное равенство удовлетворялось в узлах $x_{i}=a+i h\left(i=0,1, \ldots, n ; h=\frac{b-a}{n}\right)$. Тогда получим систему из $n+1$ уравнений

$$
\varphi_{\mathrm{III}, i}^{\prime \prime}+p\left(x_{i}\right) \quad \varphi_{\mathrm{III}, i}^{\prime}+q\left(x_{i}\right) y_{i}=r\left(x_{i}\right) \quad(i=0,1, \ldots, n)
$$

c $n+1$ неизвестными $y_{1 / s}, y_{1}, \ldots, y_{n-1}, y_{n-1 / 2}$ (так как $y_{0}=y_{n}=0$ на основании (3.2)), которые можно считать приближенными значениями точного решения $y(x)$ задачіз (3.1)-(3.2) в узлах $x_{i}$. Такой способ приближенного решения является интерполяшионным. В отличие от обыкновенного интерполяционного метода, рассмотренного в [ $\left.{ }^{2}\right]$, 
и его видоизменений, рассмотренных в [3], при этом способе приближенное решение ищется не в виде полинома, а в виде кусочно-кубической два раза дифференцируе мой функции.

Если $p(x), q(x)$ и $r(x)$ непрерывны ма $[a, b]$ и задача $(3.1)-(3.2)$ имеет единственное решение при любом $r(x)$, то система (3.3) имеет единственное решение для всех достаточно больиих $n$ и определенные этими решениями функции $F_{\text {III }}, F^{\prime}$ II $u$ $F^{\prime \prime}{ }_{\text {II }}$ равномерно сходятся на $[a, b] \kappa$ решению $y_{*}(x)$ задачи $(3.1)-(3.2)$ и к соответствующим производным этого решения со скоростью

$$
\max _{a \leqslant x \leqslant b}\left|y^{(i)}(x)-F_{\text {III }}^{(i)}[f ; x]\right|=O\left(\omega_{p}\left(\frac{1}{n}\right)+\omega_{q}\left(\frac{1}{n}\right)+\omega_{r}\left(\frac{1}{n}\right)+\frac{1}{n}\right), \quad(i=0,1,2)
$$

где $\omega_{p}, \omega_{q} u \omega_{r}-$ модули непрерывности функций $p(x), q(x)$ u $r(x)$ соответственно.

Для доказательства этого предложения пользуемся общей теорией приближенных методов Л. В. Канторовича [4].

Задачу $(3.1)-(3.2)$ рассмотрим как задачу о решении операторного уравнения

$$
G y+H y=r \text {. }
$$

где $G$ и $H\left(G y=y^{\prime \prime}, H y=p y^{\prime}+q y\right)$ - линейные операторы, отображающие пространство $y$ функций, два раза непрерывно дифференцируемых на $[a, b]$ и удовлетворяющих условиям (3.2) с нормой

$$
\|y\|=\max _{a \leqslant x \leqslant b}\left|y^{\prime \prime}(x)\right|
$$

в пространство $C$ всех непрерывных на $[a, b]$ функций с обыкновенной нормой. Система (3.3) тогда эквивалентна уравнению

$$
G y+P_{n} H y=P_{n} r
$$

где $P_{n} z=\tilde{z}-$ кусочно-линейная функция, значения которой в точках $x_{0}, x_{1}, \ldots, x_{n}$ совпадают с соответствующими значениями функции $z(x)$. Тогда

и для любого $i \cdot(i=0,1, \ldots, n-1)$

$$
\left\|P_{n}\right\|=1
$$

$$
\begin{gathered}
\max _{x i \leqslant x \leqslant x_{i+1}}|z(x)-\tilde{z}(x)|=\max _{x_{i} \leqslant x \leqslant x_{i+1}} \frac{1}{h} \mid\left[z(x)-z\left(x_{i}\right)\right]\left(x_{i+1}-x\right)+ \\
+\left[z(x)-z\left(x_{i+1}\right)\right]\left(x-x_{i}\right) \mid \leqslant \omega_{z}(h),
\end{gathered}
$$

так что

$$
\left\|z-P_{n} z\right\| \leqslant \omega_{i}(h) .
$$

Дальше известно [4], что в наших условиях

$$
|y(x)| \leqslant A\|y\|, \quad\left|y^{\prime}(x)\right| \leqslant B\|y\|,
$$

где $A$ и $B-$ постоянные, не зависящие от $y$. На основании (3.9) и (3.10) имеем

$$
\begin{gathered}
\left\|H y-P_{n} H y\right\|=\max _{a \leqslant x \leqslant b}\left|p(x) y^{\prime}(x)+q(x) y(x)-P_{n}\left[p(x) y^{\prime}(x)+q(x) y(x)\right]\right| \leqslant \\
\leq \sup _{\left|x^{\prime}-x^{\prime \prime}\right| \leqslant h}\left|p\left(x^{\prime}\right) y^{\prime}\left(x^{\prime}\right)+q\left(x^{\prime}\right) y\left(x^{\prime}\right)-p\left(x^{\prime \prime}\right) y^{\prime}\left(x^{\prime \prime}\right)-q\left(x^{\prime \prime}\right) y\left(x^{\prime \prime}\right)\right|= \\
=\sup _{\left|x^{\prime}-x^{\prime \prime}\right| \leqslant h} \mid\left[p\left(x^{\prime}\right)-p\left(x^{\prime \prime}\right)\right] y^{\prime}\left(x^{\prime}\right)+p\left(x^{\prime \prime}\right)\left[y^{\prime}\left(x^{\prime}\right)-y^{\prime}\left(x^{\prime \prime}\right)\right]+
\end{gathered}
$$




$$
\begin{aligned}
& +\left[q\left(x^{\prime}\right)-q\left(x^{\prime \prime}\right)\right] y\left(x^{\prime}\right)+i q\left(x^{\prime \prime}\right)\left[y\left(x^{\prime}\right)-y\left(x^{\prime \prime}\right)\right] \mid \leqslant \\
& \leqslant \omega_{p}(h) B\|y\|+K\|y\| h+\omega_{q}(h) A\|y\|+L B\|y\| h,
\end{aligned}
$$

где $K=\max _{a \leqslant x \leqslant b}|p(x)|$ и $L=\max _{a \leqslant x \leqslant b}|q(x)|$. Следавательно,

$$
\left\|H y-P_{n} H y\right\| \leqslant M\left(\omega_{p}(h)+\omega_{q}(h)+h\right)\|y\| .
$$

Наше предложение следует теперь из $(3.8)$, (3.9), (3.11) и из общей теории Л. В. Қанторовича.

Из доказанного предложения, в частности, следует, что

если $f(x)$ на $[a, b]$ два раза непрерывно дифференцируема и $\omega_{f \prime}(h)$ модуль непрерывности $f^{\prime \prime}(x)$, то

$$
\max _{a \leqslant x \leqslant b}\left|f(i)(x)-F_{\text {III }}^{(i)}[f ; x]\right|=O\left(\omega_{f^{\prime \prime}}\left(\frac{1}{n}\right)\right) \text { для } i=0,1,2 .
$$

\title{
Л И Т Е Р А Т У Р A
}

1 J. C. Holl a d a y, A smoothest curve approximation. Math. Tables and other Aids to Computation, Vol. XI, No. 60, 1957, 233-243.

2. Э. Б. К а рп и лов с к а я, Усп. матем. наук VIII, вып. $3(55), 1953,111-118$.

3. И. Петерсен, О сходимости приближенных методов интерполяционного типз для обыкновенных дифференциальных уравнений. Изв. АН ЭССР, Сер. физ.мат. и техн. наук, т. X, № 1, 1961.

4. Л. В. К ан торов ич, Г. П. А килов, Функциональный анализ в нормированных пространствах. М., Физматгиз, 1959.

Институт кибернетики

Академии наук Эстонской ССР

Поступила в редакцию

24. II 1961

\section{TOKITI POLUNOMIAALSEST APROKSIMATSIOONIST}

\author{
I. Petersen, \\ füüsikalis-matemaatiliste teaduste kandidaat
}

Resümee

Artiklis käsitletakse ühemuutuja funktsiooni interpoleerimist tükiti kuuppolünomiaalsete kaks korda pidevalt diferentseeruvate funktsioonidega. Tuletatakse avaldised aproksimeeriva funktsiooni esimese ja teise tuletise jaoks. Selle interpolatsioonmenetfuse alusel esitatakse harilike teist järku diferentsiaalvōrrandite rajaülesannete ligikaudse lahendamise meetod ja tõestatakse tema koonduvus.
Eesti NSV Teaduste Akadeemia
Küberneetika Instituut
Saabus toimetusse
24. II 1961

\section{OBER DIE STUCKWEISE POLYNOMISCHE APPROXIMATION}

\section{Petersen}

\section{Zusammenfassung}

In der Abhandlung wird die Interpolation der Funktionen einer Veränderlichen mit stückweise kubisch-polynomischen zweimal stetig differenzierbaren Funktionen behandelt. Es werden die Ausdrücke der ersten und zweiten Ableitung der approximierenden Funktion hergeleitet. Auf Grund dieses Interpolationsverfahrens wird eine Methode zur angenäherten Lösung der Grenzaufgaben der gewöhnlichen Differentialgleichungen zweiter Ordnung dargestellt und ihre Konvergenz bewiesen. 